\title{
Selective Retrieval of Abstract Semantic Knowledge in Left Prefrontal Cortex
}

\author{
Robert F. Goldberg, ${ }^{1}$ Charles A. Perfetti, ${ }^{2}$ Julie A. Fiez, ${ }^{2}$ and Walter Schneider ${ }^{2}$ \\ ${ }^{1}$ University of Pennsylvania, Center for Cognitive Neuroscience, Philadelphia, Pennsylvania 19104, and 2University of Pittsburgh, Center for the Neural \\ Basis of Cognition, Pittsburgh, Pennsylvania 15260
}

\begin{abstract}
Research into the representation and processing of conceptual knowledge has typically associated perceptual facts with sensory brain regions and executive retrieval mechanisms with the left prefrontal cortex. However, this dichotomy between knowledge content and retrieval processes leaves unanswered how the brain supports concepts less reliant on direct sensory experiences. We used neuroimaging methods to investigate whether an increased abstractness in semantic decisions, in contrast to increased response difficulty, is associated with increased left prefrontal activation. Participants were presented with concrete animal names and asked to verify sensory and abstract properties that corresponded only to the animal category. Candidate semantic regions were localized in left inferior, frontopolar, and dorsolateral prefrontal cortex in contrast to a pseudoword control. Activity in each of these prefrontal regions was associated with significantly increased activity for abstract relative to sensory semantic decisions, regardless of increased response difficulty and even when controlling for the response times of participants. These results suggest that more abstract, or verbally-mediated, semantic knowledge of concrete items, in contrast to more sensory-based properties, is specifically supported by the left prefrontal cortex. Semantic retrieval mechanisms may rely on abstract representations, likely coded through a verbal format, to mediate task demands when perceptual information is insufficient.
\end{abstract}

Key words: concept; declarative; decision; fMRI; language; memory

\section{Introduction}

Models of semantic memory have increasingly specified the relationship between sensory brain regions and knowledge of perceptual object properties (Martin and Chao, 2001; Goldberg et al., 2006a; Thompson-Schill et al., 2007). For instance, ventral temporal brain regions appear to support visual semantic properties, whereas more dorsal temporal and parietal regions are associated with sound and touch qualities. However, the exact contributions of the prefrontal cortex to semantic memory have yet to be determined. Typically, inferior prefrontal regions have been thought to support executive processes during active semantic retrieval (Martin and Chao, 2001). In this regard, brain regions involved in the representation of semantic content are theorized to be distinct from regions supporting the recollection of that knowledge.

Even as studies have increasingly focused on the left prefrontal cortex for its apparent functions in semantic tasks, its exact role in semantic memory remains unclear. Initially, the left inferior prefrontal cortex (LIPFC) was thought to underlie semantic retrieval or semantic working memory (Petersen et al., 1988, 1989; Kapur

Received June 5, 2006; revised Feb. 4, 2007; accepted March 1, 2007.

This work was supported by National Science Foundation Grants BCS-0121943 and PGE-9987588. We thank M. McHugo, I. Suzuki, and B. Smith for their invaluable assistance and L. Barsalou, J. McClelland, S. Thompson-Schill, R. Buckner, and two anonymous reviewers for their helpful advice and suggestions.

Correspondence should be addressed to Robert F. Goldberg, Center for Cognitive Neuroscience, 3720 Walnut Street, Philadelphia, PA 19104. E-mail: robg@psych.upenn.edu.

D0I:10.1523/JNEUROSCI.2381-06.2007

Copyright $\odot 2007$ Society for Neuroscience $\quad$ 0270-6474/07/273790-09\$15.00/0 et al., 1994; Demb et al., 1995; Fiez, 1997; Gabrieli et al., 1998). The first intensive analyses of LIPFC functioning (ThompsonSchill et al., 1997) suggest a more domain-general role in supporting the selection of task-relevant responses from among competing alternatives, which may be particularly necessary during semantic retrieval. In support of this hypothesis, numerous neuroimaging and neuropsychological studies have tested the role of the ventrolateral prefrontal cortex as competition increases during executive processing (Kan and Thompson-Schill, 2004). In contrast, Wagner et al. (2001) found that LIPFC appears to mediate controlled semantic retrieval when associate strength is weak even as selection demands were held constant.

Although attempts have been made to reconcile the selection during competition and controlled retrieval accounts (Badre and Wagner, 2002; Thompson-Schill et al., 2005), to our knowledge no study has directly manipulated semantic content with task demands to examine the role of the prefrontal cortex during semantic retrieval. The weaker associates generally used to test controlled semantic retrieval are more abstract in nature and so may require additional representations to support retrieval, whereas increased competition during retrieval may similarly involve additional demands on knowledge content. In this regard, we propose a third account of left inferior prefrontal contributions to semantic processing. Specifically, the left prefrontal cortex may selectively support abstract content during retrieval rather than strictly executive aspects of semantic processing. Recent work suggests that prefrontal retrieval mechanisms may be aided by particular representations that guide response selection 
(Miller and Cohen, 2001) with neurons in the prefrontal cortex encoding the particular abstract rule used to solve a perceptual classification (Wallis et al., 2001), even when the stimulus dimensions are continuous (Freedman et al., 2001). The left prefrontal cortex may therefore contribute not only executive retrieval processes to semantic memory but also specific content in support of those mechanisms.

To examine this hypothesis, we used semantic property verification decisions to dissociate response difficulty and semantic abstractness on activity in the left prefrontal cortex. This experiment aimed to test whether the left prefrontal cortex is involved in processing semantic content regardless of task demands. Verification of abstract semantic properties was expected to rely on the LIPFC, thus explaining the activation of this region during demanding semantic tasks in which abstractness has not been controlled. In contrast, other left prefrontal regions may help to mediate semantic control functions when task demands increase.

\section{Materials and Methods}

Participants. We obtained informed consent from 15 right-handed, native American-English speakers enrolled at the University of Pittsburgh. Using functional magnetic resonance imaging (fMRI), they were scanned while performing property verification decisions. One participant was removed from the sample because of excessive head motion $(>3 \mathrm{~mm})$ during the scanning session. Therefore, a total of 14 participants (six female, eight male; mean age, 21.87 years; range, $18-27$ years) were included in data analyses.

Stimuli and paradigm. During fMRI scanning, participants were presented with words and asked to verify whether a property belongs to the meaning of the item. To control the semantic content of each verification decision, all items and properties were drawn from the category of animals. Through behavioral norming studies, verification decisions were selected that varied on the degree of item-property abstractness and overall response difficulty. These property verification questions were controlled for how long, on average, it takes subjects to respond to the items ("difficulty") and the degree to which an item possesses the property through perceptual or verbal experiences ("abstractness"). Abstractness was therefore controlled through ratings based on the extent to which item-property relationships relied more on direct sensory experiences or indirect verbally mediated knowledge, as suggested by dualcoding accounts (Paivio, 1986). A pseudoword letter detection task was used as a baseline control in contrast to the property verifications to localize candidate semantic regions in the prefrontal cortex.

Four verification questions, which varied across item-property abstractness and response difficulty, were presented in a blocked design. Accordingly, abstractness refers to the ratings given, on a five-point scale, for item-property relationships depending on whether the relationship relies more on directly perceived experiences ("sensory") or indirectly learned verbal knowledge ("abstract"). In contrast, verification questions were also varied with regard to the response time (RT) typically required to verify the given property. Averaging across the set of items within each verification question, decisions were either generally "easy" or "hard."

One set of behavioral-only participants $(n=51 ; 14$ male, 37 female; mean age, 18.96 years) performed the property verification task on a superset of properties and items used in this experiment to identify items and properties that varied according to their response difficulty. Another set of behavioral-only participants ( $n=37 ; 12$ male, 25 female; mean age, 18.94 years) rated, on a five-point scale, the degree to which they knew the relationship between the given item (e.g., leopard, mosquito) and a potential semantic property (e.g., has fur, has bones). Participants indicated whether they knew the property relationship more from having directly experienced it (sensory) or more from having read about it or been taught it (abstract), a slight variation from the traditional instructions of lexical abstractness (Toglia and Battig, 1978). An abstract property relationship was therefore defined for the participants as one that would be typically learned indirectly through verbal facts, as suggested previously (Paivio, 1986; Noppeney and Price, 2003), whereas a sensory relationship was explained as one that could be directly perceived through experiences with the item. The behavioral data allowed for the selection of verification questions, through the items contained within them, to maximize two of the four possible extremes with regard to difficulty and abstractness: (1) easy and perceptual, "has four feet?" (average RT, 671.55 ms; abstractness, 1.71); (2) hard and perceptual, "has fur?" (average RT, $769.46 \mathrm{~ms}$; abstractness, 1.72); (3) easy and abstract, "can be trained?" (average RT, 710.63 ms; abstractness, 3.54); (4) hard and abstract, "lays eggs?" (average RT, $822.81 \mathrm{~ms}$; abstractness, 3.47).

Finally, the four examined property verification questions were matched for any possible confounding lexical factors, including letter length (average, 5.78), phonological length (average, 1.83), and lexical familiarity (average, 0.34). This latter measure is an empirically derived estimate, in contrast to frequency norms (Francis and Kucera, 1982), of how common a given word form based on $z$-score normalized response times and accuracy patterns of behavioral-only participants when the items were presented in a lexical decision experiment (R.F.G., unpublished data). Word frequency norms indirectly measure the occurrence of word forms in printed texts and thus are very likely not representative of the lexical knowledge of the tested population. In contrast, this lexical familiarity measure provides a direct estimate of lexical knowledge by testing other participants from the population in a speeded lexical decision experiment and using their response times and accuracy patterns, and the variability thereof, to control how well the particular lexical items are known. In total to date, 183 participants have been included in this behavioral-only testing. This lexical familiarity measure has been similarly used in other neuroimaging work (Goldberg et al., 2006a,b).

During the neuroimaging experiment, participants were asked to determine whether an animal possessed a given property from one of four verification decisions. These property decisions were presented in blocks with six consecutive trials of the same question. In each trial, only the stimulus word appeared on the screen, and participants had up to 1500 $\mathrm{ms}$ to verify the item-property relationship with a fixation cross after the stimulus for $1500 \mathrm{~ms}$. The verification question for each modality was presented on an instruction screen, for $8 \mathrm{~s}$, immediately before each block of six trials. An equal number of affirmative and negative items were randomly presented for each verification question and within each block of six trials. Participants were trained to respond as quickly as possible in verification decisions using a practice version of the paradigm with properties and stimuli not presented during the experiment.

The control task used to localize candidate semantic regions in the prefrontal cortex consisted of a letter detection decision (e.g., Does "warble" contain the letter "e"?). Stimuli in this control condition consisted of pronounceable pseudowords constructed from rearranged letters of a subset of the animal names used in the semantic property verifications.

Data acquisition. The protocol for this study was approved by the Institutional Review Board at the University of Pittsburgh. All MRI scanning was conducted at the Brain Imaging Research Center of the University of Pittsburgh and Carnegie Mellon University on a 3 tesla Siemens (Knoxville, TN) Allegra magnet. The scanning session began with the collection of scout, in-plane, and volume anatomical series, whereas functional scans corresponded to two runs of the experiment. The inplane structural scan served as the anatomical reference for all functional series that were collected in the same axial slices using a $\mathrm{T} 2{ }^{\star}$-weighted echo-planar imaging pulse sequence (echo time, $30 \mathrm{~ms}$; repetition time, $3000 \mathrm{~ms}$; field of view, $210 \mathrm{~mm}$; slice thickness, $3.0 \mathrm{~mm}$ with no gap between slices; flip angle, $90^{\circ}$, in-plane resolution, $\left.3.125 \mathrm{~mm}^{2}\right)$. Each trial was associated with one functional volume acquisition (repetition time). The volume anatomical scan was acquired in a sagittal plane $\left(1 \mathrm{~mm}^{3}\right)$ using the Siemens magnetization-prepared rapid-acquisition gradient echo sequence, and the functional data were coregistered to these images for analyses. Stimuli were presented and responses were collected using the E-Prime software (Psychology Software Tools, Pittsburgh, PA).

Statistical analyses. Behavioral data were examined using two-factor ANOVA tests to examine significant effects of abstractness and difficulty, as well their interaction. Paired sample $t$ tests were used to compare each of the semantic verifications with the pseudoword control.

The neuroimaging data were preprocessed and analyzed using the BrainVoyager 2000 software (Brain Innovation, Maastricht, The Nether- 
lands). Preprocessing steps included the following: six-parameter threedimensional motion-correction, slice-scan time correction using linear interpolation, voxelwise linear detrending, and spatial smoothing with an $8 \mathrm{~mm}$ full-width half-maximum Gaussian kernel. Spatial normalization was performed using the standard nine-parameter landmark method of Talairach and Tournoux (1988). A general linear model (GLM) was defined for each run that included six regressors that modeled the blood oxygenation level-dependent (BOLD) response (Boynton et al., 1996) to correct trials within each verification block and to inaccurate trials. Contrasts in the neuroimaging data were made between the verification questions based on activity differences in regions of interest (ROIs). Candidate ROIs in the prefrontal cortex were localized by contrasting all semantic verification blocks with the pseudoword letter detection task in a weighted GLM with participants as a random factor $(p<$ 0.01 , uncorrected). To examine changes in activation across these ROIs, a repeated-measures ANOVA was used with abstractness, difficulty, and region as within-subject factors and participants as a random factor. To examine the independent contributions of abstractness and difficulty, and their interaction, on activity within these prefrontal ROIs, two-factor ANOVA tests, with participant as a random factor, were conducted on the neuroimaging data based on the percentage signal change from the pseudoword control. Planned contrasts between particular verification questions were conducted with paired sample $t$ tests.

To examine regions of interest from the relevant literature that were not otherwise indicated, three-dimensional areas were defined for voxels $12 \mathrm{~mm}$ surrounding the reported peak Talairach coordinates. To initially limit the set of coordinates, we used the meta-analysis of Gold and colleagues (Gold and Buckner, 2002; Gold et al., 2005) and included other coordinates based on a sample of relevant reports in the literature that were not otherwise included (see Table 3 ). The semantic and control conditions were then examined in the present data. The posterior aspect of the left prefrontal cortex and middle temporal cortex were analyzed for semantic effects, whereas regions of the supramarginal and precentral gyri were examined for effects specific to the control condition (see Table 3).

To better control for the possibility of difficulty-based effects, quartiles were computed for each verification decision in which the 50th percentile represented the trial for the median response time for each verification decision within each neuroimaging participant. These difficulty-based quartiles were compared using two-factor ANOVA tests to examine activation within the prefrontal regions of interest. Although this eventrelated analysis is potentially problematic based on the blocked paradigm design, it is warranted post hoc for at least two reasons. First, trials were randomly distributed within each verification question allowing for the deconvolution of the BOLD response for each quartile. That is, the random "jitter" between the easier and harder trials, and the averaging of 24 trials for each quartile, allows for a more precise characterization of activation changes associated with response time. Second, although blocked effects may tend to overwhelm activation changes associated with individual trials, the signal-to-noise for individual trials would seem sufficient for this post hoc analysis provided that only a portion of the quartile comparisons prove significant. That is, if large-scale differences were found between the abstract and perceptual quartiles, it could be argued that the blocked design masks activation increases associated with individual trials. However, if differences in PFC activity are variable across quartiles and the associated verification decisions, then any comparisons that prove significant would appear more robust in accounting for between-trial variance. Indeed, although averaging across the slowest $75 \%$ of sensory trials in contrast to the fastest $75 \%$ of abstract trials shows main effects of abstractness (see Fig. 2), direct comparisons between specific quartiles show variable effects depending on the quartile involved (see Table 4).

In whole-brain analyses, main effect direct contrasts were examined using a GLM with participants as a random factor $(p<0.01$, uncorrected). Regions in these contrasts consisting of fewer than 10 contiguous voxels are not reported (see Table 5).
Table 1. Response times and accuracy rates (with SEM) for each property verification and the pseudoword control

\begin{tabular}{ccc}
\hline Condition & Response time $(\mathrm{ms})$ & Accuracy \\
\hline $\begin{array}{l}\text { Perceptual } \\
\text { Easy }\end{array}$ & $822(23)$ & \\
Hard & $875(20)$ & $0.97(0.01)$ \\
Abstract & & $0.89(0.02)$ \\
Easy & $925(25)$ & \\
Hard & $980(28)$ & $0.85(0.01)$ \\
Control & $752(27)$ & $0.78(0.02)$ \\
\hline
\end{tabular}

\section{Results}

\section{Behavioral performance}

The behavioral results indicate (Table 1 ) that the property verification decisions significantly differed with respect to overall response time and accuracy. These effects were expected given that the verification questions were normed on these stimulus dimensions in the previous behavioral studies. The two abstract questions were responded to, on average, $\sim 11 \%$ less accurately than the perceptual verifications $\left(F_{(1,13)}=52.62 ; p<0.001\right)$. Responses to the two hard questions were $\sim 7 \%$ less accurate than for the easy questions $\left(F_{(1,13)}=19.28 ; p<0.001\right)$. There was no significant interaction between abstractness and difficulty in the accuracy of the decisions. The response time data for correct trials also show main effects for abstractness and difficulty but no interaction. Participants responded $\sim 110 \mathrm{~ms}$ slower for the abstract than for the perceptual questions $\left(F_{(1,13)}=22.22 ; p<\right.$ 0.001 ) but responded $\sim 60$ ms more slowly for the hard than for the easy verification decisions $\left(F_{(1,13)}=4.95 ; p<0.05\right)$. Response times to the pseudoword letter detection were significantly faster than to any of the semantic properties ( $t$ values $>5.06$; $p$ values $<0.001$ ) with accuracy significantly better ( $t$ values $>3.87$; $p$ values $<0.001)$ than for all of the semantic decisions except for the easy perceptual verification.

\section{Neuroimaging data}

By contrasting the semantic property verifications with the pseudoword control task, candidate semantic regions were localized ( $p<0.01$, uncorrected) to independently investigate the effects of abstractness and response difficulty. This comparison yielded four prefrontal ROIs in the left hemisphere (Table 2). The across-region analyses showed a main effect of abstractness $\left(F_{(1,13)}=28.486 ; p<0.001\right)$ with trends for the main effect of region $\left(F_{(1,13)}=3.528 ; p=0.083\right)$ and the three-way interaction between region, abstractness, and difficulty $\left(F_{(1,13)}=3.694 ; p=\right.$ 0.077). This trend for the three-way interaction appears to be driven by the increased response in lateral Brodmann area (BA) 47 , in contrast to medial BA 47, for the easier abstract decision $\left(t_{(13)}=3.01 ; p<0.01\right)$. No other effects across these prefrontal regions of interest were observed.

The semantic decisions, in contrast to the pseudoword letter detection, were associated with increased activation in two anterior regions of the left inferior prefrontal cortex (aLIPFC) within BA 47. A medial aspect of the aLIPFC (Fig. $1 A$ ) showed increased activation for the abstract verifications by over the perceptual properties $\left(F_{(1,13)}=11.82 ; p=0.001\right)$. This region showed neither a significant effect of response difficulty nor an interaction between difficulty and verification abstractness. In contrast, a more lateral aspect of the aLIPFC (Fig. $1 B$ ) showed increased activation for the abstract decisions $\left(F_{(1,13)}=57.39 ; p<0.001\right)$ as well as a significant interaction with response difficulty $\left(F_{(1,13)}=\right.$ $14.11 ; p<0.001)$. This latter finding appears to be specifically 
Table 2. Candidate semantic regions in the left hemisphere showing $(p<0.01$, uncorrected) increased activation for the semantic decisions in contrast to the pseudoword control

\begin{tabular}{|c|c|c|c|c|c|c|}
\hline \multirow[b]{2}{*}{ Region } & \multirow[b]{2}{*}{$B A$} & \multicolumn{3}{|c|}{ Talairach coordinates } & \multirow[b]{2}{*}{ Voxel count } & \multirow[b]{2}{*}{$p$ value } \\
\hline & & $x$ & $y$ & $z$ & & \\
\hline Medial inferior prefrontal cortex & 47 & -33 & 33 & 1 & 13 & 0.0050 \\
\hline Lateral inferior prefrontal cortex & 47 & -45 & 35 & 0 & 29 & 0.0032 \\
\hline Dorsolateral prefrontal cortex & 9 & -48 & 24 & 31 & 43 & 0.0045 \\
\hline Frontopolar cortex & 10 & -33 & 50 & 19 & 52 & 0.0052 \\
\hline
\end{tabular}

driven by increased activation to the easier abstract verification in contrast to the more difficult abstract property decision $\left(t_{(13)}=\right.$ 5.83; $p<0.001)$.

The functional contrast between the property decisions and the letter detection control also yielded two additional regions in more frontal and dorsal aspects of the left prefrontal cortex, specifically in frontopolar cortex (FPC) (BA 10) and dorsolateral prefrontal cortex (DLPFC) (BA 9). These regions similarly showed significantly increased activity for the abstract properties relative to the sensory-based verifications. Activity in the FPC (Fig. 1C) indicates that the abstract verifications, on average, led to a significant increase of $0.2 \%$ in contrast to the perceptual verifications $\left(F_{(1,13)}=14.26 ; p<0.001\right)$. The significant interaction $\left(F_{(1,13)}=4.55 ; p<0.05\right)$ between abstractness and response time in this region appears to be primarily driven by an increased response to the harder perceptual decision in contrast to the easier perceptual verification $\left(t_{(13)}=1.88\right.$; $\left.p<0.05\right)$. The DLPFC (Fig. 1D) showed a similar response to the abstractness of the verification questions $\left(F_{(1,13)}=18.81 ; p<0.001\right)$ but less clearly to the overall response difficulty.

Posterior regions of the left inferior prefrontal cortex, including BA 44 and 45, and left middle temporal cortex (BA 21) were not localized in the functional contrast, even at less conservative thresholds ( $p<0.15$, uncorrected). To examine activation in these areas, we used coordinates from reports in the literature to determine whether these regions showed effects specific to the semantic conditions. As shown in Table 3, the results indicate that increased activation for the semantic conditions, in contrast to the pseudoword control, in these areas were not robust if apparent. The effects observed in regions representative of BA 44 approached significance (uncorrected), but this effect appears because of a slight overlap of these areas with the DLPFC (BA 9) region indicated in the functional contrast, although the effect weakens as the coordinates of the BA 44 regions and $\mathrm{BA} 9$ region diverge. Regions in the middle temporal cortex showed no increased activation for the semantic conditions, whereas one region was associated with increased activation for the control condition. Precentral and supramarginal regions previously indicated as specifically active for pseudoword conditions (Gold and Buckner, 2002, Gold et al., 2005), in contrast to semantic tasks, were likewise indicated in this dataset ( $t$ values $>3.35$; $p$ values $<0.01$ ).

Even as the difficulty manipulation for the verification questions was replicated in the response profiles of the neuroimaging participants, nonlinear, and still latency-based, activity changes in the PFC could explain the differences between the abstract and perceptual decisions. To rule out this interpretation, response times for individual trials within the neuroimaging participants were used to create bins associated with the 25, 50, 75, and 100\% latency quartiles of each decision. By matching the $75 \%$ slowest responses for the sensory verifications and the $75 \%$ fastest responses for the abstract decisions, the two types of verification questions were more directly controlled for increased difficulty. Based on participant-specific response times, in this subset of trials, the more sensory-based decisions (mean \pm SE RT, $913 \pm$ $25 \mathrm{~ms}$ ) are slower than the abstract questions (mean \pm SE RT, $866 \pm 22 \mathrm{~ms}$ ) even as the difference in the abstractness rating remains (sensory, 2.1; abstract, 3.1). The results indicate that, controlling for response times to individual trials, increased semantic abstractness is strongly associated with increased activation across the four PFC regions of interest (Fig. 2, Table 4). In the left ventral prefrontal regions (BA 47), the medial $\left(F_{(1,13)}=6.69\right.$; $p=0.012)$ and lateral $\left(F_{(1,13)}=37.86 ; p<0.001\right)$ aspects were associated with significantly increased activation for the abstract property verifications relative to the sensory-based decisions. These significant differences based on item-property abstractness, and controlling for the response times of the participants, are also apparent in the frontopolar $\left(F_{(1,13)}=7.62 ; p<0.01\right)$ and dorsolateral $\left(F_{(1,13)}=9.22 ; p<0.01\right)$ regions.

The whole-brain direct contrast between abstract and perceptual semantic properties ( $p<0.01$, uncorrected) shows leftlateralized increased activation for the abstract property verifications in parietal, temporal, and occipital regions in addition to the prefrontal cortex (Fig. 3, Table 5). Less extensive activation was found for perceptual relative to abstract semantic property verifications in right parietal and ventral occipital regions. The whole-brain direct contrast between the easy and hard decisions $(p<0.01$, uncorrected) indicated one region in the left lateral temporal cortex more responsive to the harder decisions, whereas multiple regions, including the left anterior temporal cortex, bilateral inferior parietal cortex, and left medial and lateral frontal cortex, were more responsive to the easier than to the more difficult decisions (Table 5). Examination of this set of regions derived from these whole-brain contrasts indicates that a subset of the semantic verifications elicited less activation than the pseudoword control explaining why these areas did not appear as candidate semantic regions in the functional localizer. In this regard, it is not clear whether these areas specifically support semantic processing or more domain-general retrieval mechanisms.

\section{Discussion}

This study examined the role of the prefrontal cortex in supporting abstract semantic decisions as response difficulty increases. The results suggest that the left prefrontal cortex is specialized to process abstract, or verbally mediated, information about concrete objects. The anterior LIPFC (BA 47) showed increased activation for abstract, in contrast to perceptual, semantic properties without a commensurate increase for more demanding decisions. More dorsal prefrontal regions, including the DLPFC (BA 9) and FPC (BA 10), also appear to be associated with semantic property verification as abstractness increases and sensitive to the difficulty of sensory semantic decisions. This delineation of prefrontal regions involved in aspects of abstract semantic pro- 
cessing is consistent with recent accounts of rule-based mechanisms (Bunge, 2004; Bunge et al., 2005) and suggests that these regions are specialized to represent and process abstract semantic knowledge within response selection mechanisms that bias posterior representations (Miller and Cohen, 2001).

Previous studies that have found left prefrontal involvement in semantic tasks may not have sufficiently controlled for the abstractness of the stimulus materials or the specific decisions required. In examining general retrieval mechanisms, these reports may have confounded content with process, even as fully dissociating the two remains problematic. For instance, the controlled retrieval account (Wagner et al., 2001) begs the question of whether a particular type of semantic information is more likely to require this hypothesized semantic function. Association norms are likely to include more abstract relationships, and the requisite representations to mediate them, as associate strength weakens. Indeed, Badre et al. (2005) manipulated associative strength and identified an anterior and ventral locus of controlled semantic retrieval that appears to converge with the left inferior prefrontal regions (BA 47) identified in the current study. That is, weaker semantic associates are more likely to rely on other linguistic representations and so controlled semantic processing may require additional abstract, and verbally mediated, semantic content. In support of this interpretation, Noppeney and Price across a number of studies (Noppeney and Price, 2002, 2003, 2004) have examined how abstract semantic knowledge relies on brain regions more generally associated with distinct semantic categories and retrieval mechanisms and depending on task and stimulus factors. Noppeney and Price (2003) identified the ventrolateral prefrontal area as indicative of a common semantic system and sensitive to verbal facts relative to a baseline condition. More recently, Noppeney and Price (2004) replicated the role of the ventrolateral prefrontal cortex with decisions involving abstract concepts (e.g., fame) relative to perceptual semantic comparisons. In this regard, semantic content more likely coded through a verbal format may specifically rely on the left prefrontal cortex to coordinate semantic retrieval and as perceptual information is insufficient to resolve task demands.

The relationship between semantic processing and domaingeneral mechanisms may be more direct than is generally assumed but based on the specifics of the knowledge involved. Although we included items only with a clear affirmative or neg-

D
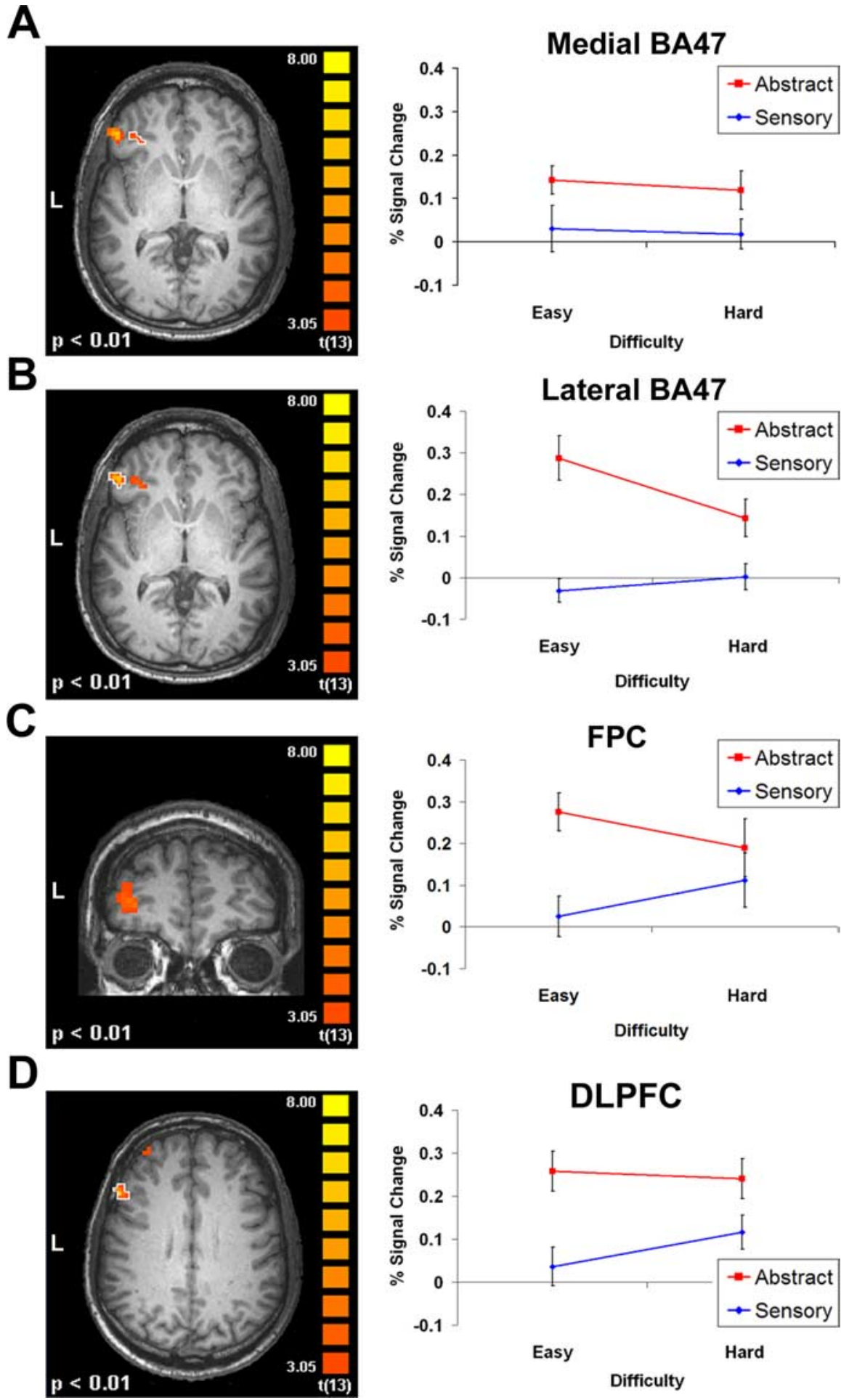

Figure 1. To examine the variability of prefrontal activity specific to semantic retrieval across these conditions, property verifications were contrasted with a pseudoword letter detection task. Activity in the aLIPFC. $\boldsymbol{A}$, The medial region is more active for abstract property verifications than for sensory-based knowledge. $\boldsymbol{B}$, In contrast, a more lateral region shows a main effect of abstractness and an interaction with response difficulty. Activity in the frontodorsal regions of the left prefrontal cortex. C, The FPC shows greater activity for abstract semantic retrieval than for the perceptual properties with an interaction based on response difficulty. D, The DLPFC shows increased activation for the abstract property verifications. Error bars represent SEM.

ative response, more borderline cases may specifically reflect the necessity of abstract semantic representations to resolve the ambiguity. For instance, prefrontal regions may be especially sensitive not only to the mediation of associative strength (Wagner et al., 2001) but also the binding of the requisite representations to 
Table 3. Regions of interest in the left hemisphere defined from previous reports to examine percentage signal change (with SEM) for the pseudoword and semantic conditions

\begin{tabular}{|c|c|c|c|c|c|c|}
\hline Report $^{a}$ & $\mathrm{ROI}^{b}$ & Atlas coordinates $^{c}$ & Pseudoword & Semantic & $t$ & $p$ \\
\hline \multirow[t]{4}{*}{1,2} & BA45 & $-47,17,24$ & $-0.052(0.025)$ & $0.018(0.031)$ & 2.170 & 0.049 \\
\hline & BA21 & $-51,-55,2$ & $-0.011(0.030)$ & $0.005(0.037)$ & 0.150 & 0.883 \\
\hline & BA40 & $-41,-43,34$ & $0.062(0.017)$ & $-0.016(0.023)$ & -3.412 & 0.005 \\
\hline & BA 6 & $-55,-1,28$ & $0.091(0.026)$ & $-0.018(0.031)$ & -3.353 & 0.005 \\
\hline \multirow[t]{2}{*}{3} & BA45 & $-46,24,12$ & $-0.007(0.027)$ & $0.007(0.025)$ & 0.449 & 0.669 \\
\hline & BA21 & $-54,-50,-18$ & $0.090(0.047)$ & $-0.031(0.064)$ & -2.075 & 0.058 \\
\hline 4 & BA44 & $-44,15,22$ & $-0.033(0.020)$ & $0.012(0.028)$ & 1.849 & 0.087 \\
\hline \multirow[t]{2}{*}{5} & BA44 & $-51,15,33$ & $-0.090(0.050)$ & $0.014(0.029)$ & 1.829 & 0.090 \\
\hline & BA21 & $-48,-48,3$ & $0.026(0.012)$ & $-0.013(0.022)$ & -2.837 & 0.014 \\
\hline
\end{tabular}

a Selected reference papers that motivate regions of interest: 1, Gold and Buckner, 2002; 2, Gold et al., 2005; 3, Noppeney and Price, 2003; 4, Thompson-Schill et al., 1999 ; and 5, Badre et al., 2005.

${ }^{b}$ ROIs are named based the nearest BA in the Talairach and Tournoux (1988) atlas.

'Coordinates of the ROl in the Talairach and Tournoux (1988) atlas.

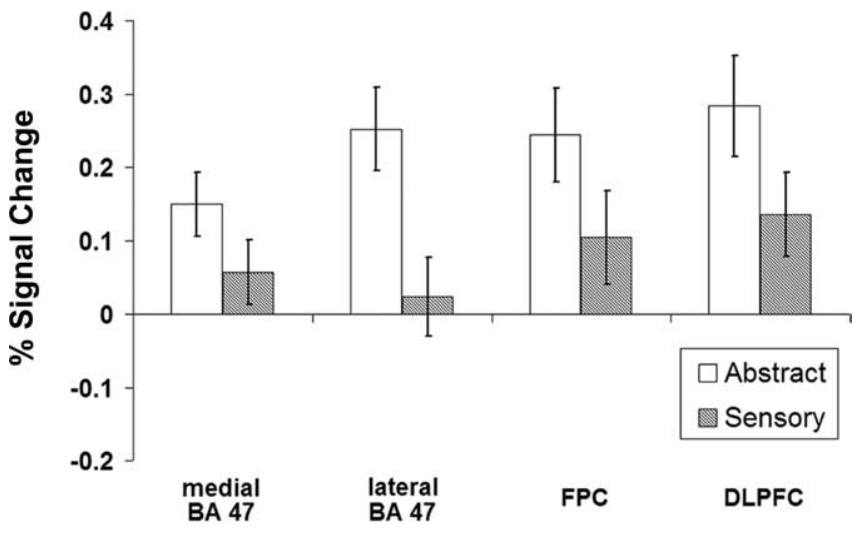

Figure 2. Segregating trials into latency-based quartiles allowed for a more directed matching of the verification questions. Comparing the $75 \%$ slowest sensory trials against the $75 \%$ fastest abstract decisions shows main effects of abstractness across the left prefrontal regions of interest. Error bars represent SEM.

modulate the associations (Bunge, 2004). Although post hoc analysis of the differences between the included properties is merely suggestive, the "lay eggs" verification by definition includes only a binary choice set. In contrast, whether an animal "can be trained" appears to be ambiguous depending on the particular item involved (e.g., tiger vs hyena) and the additional semantic information (e.g., circus, hoop, whip) necessary to resolve the decision. The prefrontal cortex may then selectively bind abstract semantic information as indicative for other domains (Ramnani and Owen, 2004) and for the integration of word meanings during analogical reasoning (Bunge et al., 2005). This interpretation is consistent with evidence showing that the prefrontal cortex is specifically involved in the evaluation of internally generated information (Christoff and Gabrieli, 2000) as well as the mediation of rule learning (Strange et al., 2001). The prefrontal cortex would therefore seem ideally suited to the verbal mediation of knowledge retrieval. More dorsal aspects of the prefrontal cortex are consistently associated with the mediation of perceptual information (Christoff and Gabrieli, 2000), and so semantic processing may co-opt these more domain-general functions when the information set is sufficiently constrained.

Posterior regions of the LIPFC, including BA 44 and 45, were not localized for the semantic conditions relative to the pseudoword control even at less conservative thresholds. Furthermore, regions defined from the literature suggest that these areas of the prefrontal cortex, and well as middle temporal regions, were not specifically active for the semantic conditions relative to the pseudoword control. As the previous literature for
LIPFC functioning suggests (Gold and Buckner, 2002; Kan and Thompson-Schill, 2004; Badre et al., 2005), the phonological representations associated with the pronounceable pseudoword controls were likely involved in the resolution of the letter detection task to a similar extent as those involved in resolving the semantic property verifications. Determining whether the given letter was present likely required controlled processing of phonology and orthography in line with the task demands associated with resolving properties of semantic knowledge. In support of this interpretation, areas more biased to phonological processing (Gold and Buckner, 2002; Gold et al., 2005), including regions of the precentral and supramarginal gyri, were seen to be specifically activated by the control task. In contrast, reports that indicate anterior LIPFC activation during phonological processing (Gold and Buckner, 2002; Clark and Wagner, 2003) suggest a role for verbally mediated processing even with nonsemantic content. Through systematically manipulating the degree of perceptual and verbally mediated information associated with pseudoword stimuli and task demands, it may be possible to demonstrate similar left prefrontal activation patterns, despite the lack of strictly semantic knowledge, as those shown here.

The lack of semantic activation in posterior cortex is somewhat surprising given the increasing number of reports indicating an association between sensory brain regions and perceptual knowledge (Goldberg et al., 2006a; Thompson-Schill et al., 2007). The whole-brain direct contrasts based on abstractness and difficulty suggest a few possible interpretations even as the observed activation differences did not appear specific to semantic knowledge. One possibility is that this null result of posterior involvement is related to the particular category tested. That is, items and properties of the tested animal category (e.g., hyena $\rightarrow$ four legs? fur?) may rely more on verbally mediated knowledge than categories examined in sensory-based reports (Goldberg et al., 2006a), such as materials (soft?) and fruits (sweet?), in which both the items and the properties are more likely to be directly experienced. Future reports will need to examine how specific categories, and their constituent members, vary based on the particular configurations of sensory and abstract properties and therefore how posterior and prefrontal regions coordinate to resolve semantic decisions as task demands and the requisite content vary. Abstract semantic knowledge may also be built atop sensory mechanisms through which the verbally mediated facts are accessed. Posterior areas, in parietal and temporal cortex, were activated more strongly for the abstract decisions in contrast to the sensory verifications, even as this increased response did not appear to be specific for semantic information. To access more abstract knowledge, it may be necessary to initially process 
Table 4. Quartile comparisons between the sensory and abstract trials, averaged across the two decisions of each, based on the response times of the neuroimaging participants

\begin{tabular}{|c|c|c|c|c|c|c|}
\hline Quartiles & Response time (ms) & Abstractness & Medial BA 47 & Lateral BA 47 & FPC & DLPFC \\
\hline \multicolumn{7}{|l|}{ Abstract } \\
\hline $25 \%$ & $730(16)$ & $3.00(0.07)$ & $0.097(0.04)$ & $0.199(0.06)$ & $0.205(0.06)$ & $0.207(0.06)$ \\
\hline $50 \%$ & $864(22)$ & $3.20(0.06)$ & $0.151(0.05)$ & $0.266(0.06)$ & $0.239(0.06)$ & $0.300(0.07)$ \\
\hline $75 \%$ & 1004 (29) & $3.09(0.06)$ & $0.196(0.04)$ & $0.291(0.06)$ & $0.288(0.07)$ & $0.346(0.07)$ \\
\hline $100 \%$ & $1248(36)$ & $3.24(0.08)$ & $0.247(0.06)$ & $0.344(0.05)$ & $0.352(0.07)$ & $0.473(0.07)$ \\
\hline \multicolumn{7}{|l|}{ Sensory } \\
\hline $25 \%$ & $671(15)$ & $2.01(0.05)$ & $0.007(0.04)$ & $-0.041(0.05)$ & $0.000(0.06)$ & $0.053(0.07)$ \\
\hline $50 \%$ & 770 (19) & $2.16(0.05)$ & $0.034(0.06)$ & $-0.007(0.05)$ & $0.003(0.07)$ & $0.037(0.05)$ \\
\hline $75 \%$ & $875(24)$ & $2.08(0.05)$ & $0.039(0.04)$ & $-0.039(0.05)$ & $0.084(0.06)$ & $0.107(0.07)$ \\
\hline $100 \%$ & $1093(32)$ & $2.10(0.05)$ & $0.098(0.04)$ & $0.116(0.06)$ & $0.227(0.06)$ & $0.262(0.05)$ \\
\hline
\end{tabular}

In Figure 2, the fastest $75 \%$ of the abstract trials ( 25,50 , and 75 quartiles) are matched based on the mean response times and directly compared against the slowest $75 \%$ of the sensory trials ( 50,75 , and $100 \%$ quartiles). For the prefrontal regions of interest, percentage signal change from the pseudoword condition is presented. SEM for each measure is given in parentheses.

sensory-based representations, as suggested by seminal stage-based models of semantic memory (Rips et al., 1973; McCloskey and Glucksberg, 1979). In this regard, activation of verbally mediated facts may involve the suppression of irrelevant sensory content especially as competition arises between conflicting semantic representations. These hypotheses point toward future studies that examine the time course of semantic processing between prefrontal cortex and regions of posterior cortex thought to support perceptual semantic properties (Noppeney and Price, 2003; Goldberg et al., 2006a). Comparisons across different categories of objects, involving sensory and abstract knowledge, may specifically dissociate into prefrontal and posterior regions, and their interaction, based on the task demands and the

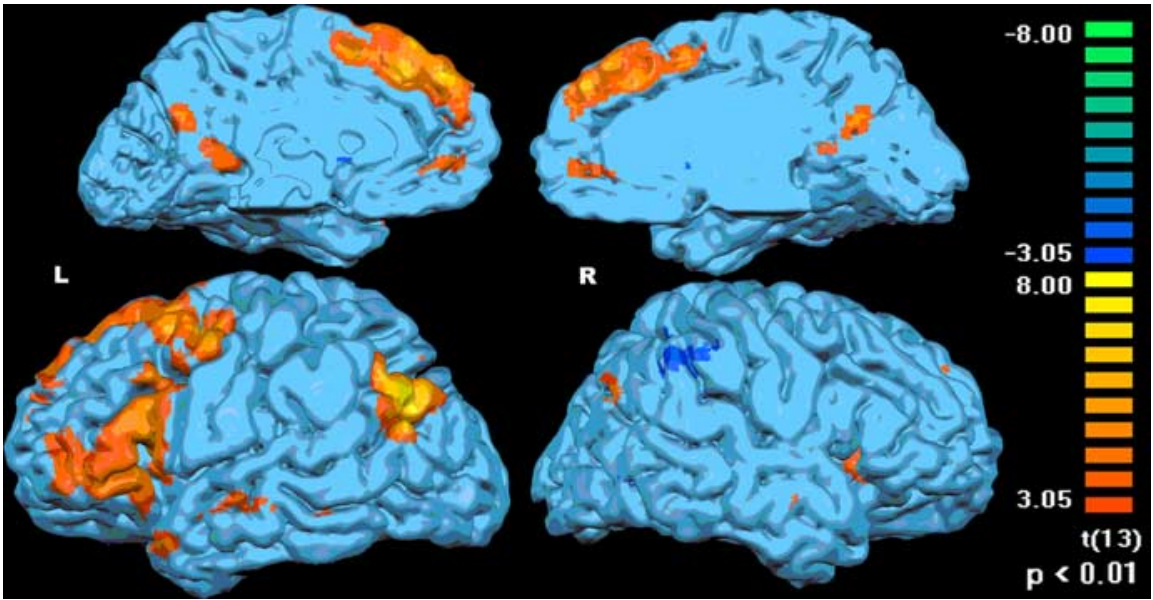

Figure 3. The whole-brain main effect contrast ( $p<0.01$, uncorrected) between the abstract and perceptual property verifications shows left-lateralized activation $(\mathrm{L})$ in the frontal, temporal, and parietal lobes for verification of abstract properties of animals and in the right parietal lobe (R) for perceptual semantic decisions. specific knowledge content required.

One intriguing interpretation of the current results is that the regions involved during abstract knowledge encoding are reactivated during retrieval, as has been suggested for the retrieval of perceptual semantic knowledge after initial encoding in sensory brain mechanisms (Barsalou, 1999). For instance, left inferior prefrontal cortex shows increased activation during semantic encoding relative to nonsemantic encoding (Demb et al., 1995), whereas activity in the LIPFC during encoding predicts later recall (Wagner et al., 1997). Considering that single-unit recording studies indicate that, after acquisition, abstract information specifically relies on the prefrontal cortex (Miller et al., 2003), semantic content more likely encoded in a verbal, or propositional, format may selectively depend on the prefrontal cortex during the later resolution of semantic decisions. Rule use has been associated more generally with the ventrolateral PFC, and current work aims to dissociate aspects of rule maintenance, retrieval, and storage within the prefrontal cortex (Bunge, 2004). Such models of prefrontal functioning during symbolic manipulation are likely to be highly informative with respect to the representation and processing of semantic knowledge. However, even as abstract semantic processing may involve explicit properties and their relationships within the language system, the neural instantiation of this knowledge need not be symbolic (Rougier et al., 2005).

A more general difficulty with examining abstract knowledge is the many ways it can be parsed based on the particular concepts involved. Cortical regions more sensitive to semantic abstractness are likely to vary depending on how it is defined and manipulated. Barsalou (2003) has recently identified at least six possible senses of abstraction and the ways in which it can be described. According to these senses, abstract properties of concrete items, of the type examined in this study, may arise from category relationships (e.g., mammals have fur), the behavioral tendency to generalize commonalities across items (e.g., central tendencies of category members), or stored representations in memory that may or may not be strictly categorical (e.g., things you take to the beach). In contrast, abstract properties may be also bound more tightly to the context in which they are generated and require the particular events or circumstances to recall the knowledge (Barsalou and Wiemer-Hastings, 2005). These contextual relationships are more likely mediated through linguistic knowledge rather than stored as language-specific representations. Although the fractionation of prefrontal functions during semantic processing is still emerging, the present results suggest that, by studying a particular semantic class in depth, namely one with an inherent hierarchical structure, this division of semantic labor is likely to become more apparent.

In summary, the prefrontal cortex appears to support the verification of abstract semantic knowledge to concrete items. By restricting semantic decisions to one superordinate category while varying the types of properties to be examined, the results suggest that subtle feature differences are associated with robust differences in activation within the prefrontal cortex. These find- 
Table 5. Results of the whole-brain direct main effect contrasts ( $p<0.01$, uncorrected) for abstractness and difficulty

\begin{tabular}{|c|c|c|c|c|c|c|}
\hline \multirow[b]{2}{*}{ Region } & \multirow[b]{2}{*}{$B A$} & \multicolumn{3}{|c|}{ Talairach coordinates } & \multirow[b]{2}{*}{ Voxel count } & \multirow[b]{2}{*}{$p$ value } \\
\hline & & $x$ & $y$ & $z$ & & \\
\hline \multicolumn{7}{|l|}{ Abstract-perceptual } \\
\hline Left frontal lobe & & -25 & 24 & 35 & 2310 & 0.002624 \\
\hline Left inferior parietal cortex & & -41 & -69 & 31 & 566 & 0.002068 \\
\hline Left posterior cingulate & 23 & -5 & -51 & 13 & 223 & 0.003805 \\
\hline Right insula & 13 & 31 & 23 & 4 & 73 & 0.004415 \\
\hline Right angular gyrus & 39 & 44 & -70 & 31 & 54 & 0.004792 \\
\hline Anterior cingulate gyri & 32 & 0 & 48 & 3 & 50 & 0.004823 \\
\hline Left middle temporal gyrus & 21 & -59 & -44 & -1 & 47 & 0.005522 \\
\hline Right superior frontal gyrus & 6 & 18 & 18 & 51 & 42 & 0.004503 \\
\hline Left middle temporal gyrus & 21 & -59 & -8 & -6 & 39 & 0.005497 \\
\hline Left parahippocampal gyrus & 36 & -30 & -31 & -14 & 25 & 0.005422 \\
\hline Left thalamus & & -8 & -13 & 10 & 15 & 0.005984 \\
\hline Left cingulate gyrus & 31 & -4 & -40 & 33 & 12 & 0.005094 \\
\hline \multicolumn{7}{|l|}{ Perceptual-abstract } \\
\hline Right inferior parietal cortex & 40 & 57 & -37 & 40 & 87 & 0.004623 \\
\hline Right precuneus & 7 & 24 & -59 & 41 & 38 & 0.004752 \\
\hline Right precuneus & 7 & 12 & -62 & 45 & 29 & 0.005399 \\
\hline Right parietal cortex & 40 & 41 & -47 & 45 & 29 & 0.005717 \\
\hline \multicolumn{7}{|l|}{ Hard-easy } \\
\hline Left inferior temporal cortex & 37 & -51 & -58 & -15 & 11 & 0.004098 \\
\hline \multicolumn{7}{|l|}{ Easy-hard } \\
\hline Left anterior temporal cortex & 21 & -48 & -7 & -12 & 63 & 0.003959 \\
\hline Left anterior temporal cortex & 38 & -61 & 14 & -10 & 16 & 0.004669 \\
\hline Left inferior parietal cortex & 39 & -41 & -64 & 22 & 38 & 0.004873 \\
\hline Left medial frontal cortex & $9 / 10$ & -7 & 57 & 34 & 30 & 0.003638 \\
\hline Left superior frontal gyrus & 6 & -10 & 16 & 56 & 36 & 0.003977 \\
\hline Right inferior parietal cortex & 39 & 48 & -68 & 13 & 41 & 0.005314 \\
\hline
\end{tabular}

ings suggest that previous accounts that have examined executive semantic mechanisms may not have sufficiently controlled the abstractness of the decisions or the stimulus materials. The left prefrontal cortex would seem to contribute the property knowledge, likely thorough a verbal format, more necessary for some semantic decisions and including areas previously thought to instantiate strictly control functions associated with semantic retrieval. Conceptual knowledge appears to consist of a network of brain regions specialized to represent perceptual and abstract knowledge content respectively. Verbally mediated knowledge may specifically aid in coordinating retrieval mechanisms in biasing posterior representations.

\section{References}

Badre D, Wagner AD (2002) Semantic retrieval, mnemonic control, and prefrontal cortex. Behav Cogn Neurosci Rev 1:206-218.

Badre D, Poldrack RA, Pare-Blagoev EJ, Insler RZ, Wagner AD (2005) Dissociable controlled retrieval and generalized selection mechanisms in ventrolateral prefrontal cortex. Neuron 47:907-918.

Barsalou LW (1999) Perceptual symbol systems. Behav Brain Sci 22:577-660.

Barsalou LW (2003) Abstraction in perceptual symbol systems. Philos Trans R Soc Lond B Biol Sci 358:1177-1187.

Barsalou LW, Wiemer-Hastings K (2005) Situating abstract concepts. In: Grounding cognition: the role of perception and action in memory, language, and thought (Pecher D, Zwaan RA, eds), pp 129-163. New York: Cambridge UP.

Boynton GM, Engel SA, Glover GH, Heeger DJ (1996) Linear systems analysis of functional magnetic resonance imaging in human V1. J Neurosci 16:4207-4221.

Bunge SA (2004) How we use rules to select actions: a review of evidence from cognitive neuroscience. Cogn Affect Behav Neurosci 4:564-579.

Bunge SA, Wendelken C, Badre D, Wagner AD (2005) Analogical reasoning and prefrontal cortex: evidence for separable retrieval and integration mechanisms. Cereb Cortex 15:239-249.

Christoff K, Gabrieli JDE (2000) The frontopolar cortex and human cogni- tion: evidence for a rostrocaudal hierarchical organization within the human prefrontal cortex. Psychobiology 28:168-186.

Clark D, Wagner AD (2003) Assembling and encoding word representations: fMRI subsequent memory effects implicate a role for phonological control. Neuropsychologia 41:304-317.

Demb JB, Desmond JE, Wagner AD, Vaidya CJ, Glover GH, Gabrieli JD (1995) Semantic encoding and retrieval in the left inferior prefrontal cortex: a functional MRI study of task difficulty and process specificity. J Neurosci 15:5870-5878.

Fiez JA (1997) Phonology, semantics, and the role of the left inferior prefrontal cortex. Hum Brain Mapp 5:79-83.

Francis WN, Kucera H (1982) Frequency analysis of English usage. Boston: Houghton Mifflin.

Freedman DJ, Riesnhuber M, Poggio T, Miller EK (2001) Categorical representation of visual stimuli in the primate prefrontal cortex. Science 291:312-316.

Gabrieli JD, Poldrack RA, Desmond JE (1998) The role of left prefrontal cortex in language and memory. Proc Natl Acad Sci USA 95:906-913.

Gold BT, Buckner RL (2002) Common prefrontal regions coactivate with dissociable posterior regions during controlled semantic and phonological tasks. Neuron 35:803-812.

Gold BT, Balota DA, Kirchhoff BA, Buckner RL (2005) Common and dissociable activation patterns associated with controlled semantic and phonological processing: evidence from fMRI adaptation. Cereb Cortex 15:1438-1450.

Goldberg RF, Perfetti CA, Schneider W (2006a) Perceptual knowledge retrieval activates sensory brain regions. J Neurosci 26:4917-4921.

Goldberg RF, Perfetti CA, Schneider W (2006b) Distinct and common cortical activations for multimodal semantic categories. Cogn Affect Behav Neurosci 6:214-222.

Kan IP, Thompson-Schill SL (2004) Selection from perceptual and conceptual representations. Cogn Affect Behav Neurosci 4:466-482.

Kapur S, Rose R, Liddle PF, Zipursky RB, Brown GM, Stuss D, Houle S, Tulving E (1994) The role of the left prefrontal cortex in verbal processing: semantic processing or willed action? NeuroReport 5:2193-2196.

Martin A, Chao LL (2001) Semantic memory and the brain: Structure and processes. Curr Opin Neurobiol 11:194-201. 
McCloskey M, Glucksberg S (1979) Decision processes in verifying category membership statements: implications for models of semantic memory. Cogn Psych 11:1-37.

Miller EK, Cohen JD (2001) An integrative theory of prefrontal cortex function. Annu Rev Neurosci 24:167-202.

Miller EK, Nieder A, Freedman DJ, Wallis JD (2003) Neural correlates of categories and concepts. Curr Opin Neurobiol 13:198-203.

Noppeney U, Price C (2002) Retrieval of visual, auditory, and abstract semantics. NeuroImage 15:917-926.

Noppeney U, Price CJ (2003) Functional imaging of the semantic system: retrieval of sensory-experienced and verbally learned knowledge. Brain Lang 84:120-133.

Noppeney U, Price C (2004) Retrieval of abstract semantics. NeuroImage 22:164-170.

Paivio A (1986) Mental representations: a dual coding approach. New York: Oxford UP.

Petersen SE, Fox PT, Posner MI, Mintun M, Raichle ME (1988) Positron emission tomographic studies of the cortical anatomy of single-word processing. Nature 331:585-589.

Petersen SE, Fox PT, Posner MI, Mintun M, Raichle ME (1989) Positron emission tomographic studies of the processing of single words. J Cogn Neurosci 1:153-170.

Ramnani N, Owen AM (2004) Anterior prefrontal cortex: Insights into function from anatomy and neuroimaging. Nat Rev Neurosci 5:184-194.

Rips LJ, Shoben EJ, Smith EE (1973) Semantic distance and the verification of semantic relations. J Verbal Learn Verbal Behav 12:1-20.

Rougier NP, Noelle DC, Braver TS, Cohen JD, O’Reilly RC (2005) Prefron- tal cortex and the flexibility of cognitive control: Rules without symbols. Proc Natl Acad Sci USA 102:7338-7343.

Strange BA, Henson RNA, Friston KJ, Dolan RJ (2001) Anterior prefrontal cortex mediates rule learning in humans. Cereb Cortex 11:1040-1046.

Talairach J, Tournoux P (1988) A co-planar stereotaxic atlas of the human brain. New York: Thieme Medical.

Thompson-Schill SL, D’Esposito M, Aguirre GK, Farah MJ (1997) Role of left inferior prefrontal cortex in retrieval of semantic knowledge: a reevaluation. Proc Natl Acad Sci USA 94:14792-14797.

Thompson-Schill SL, D’Esposito M, Kan IP (1999) Effects of repetition and competition on activity in left prefrontal cortex during word generation. Neuron 23:513-522.

Thompson-Schill SL, Bedny M, Goldberg RF (2005) The frontal lobes and the regulation of mental activity. Curr Opin Neurobiol 15:219-224.

Thompson-Schill SL, Kan IP, Oliver RT (2006) Functional neuroimaging of semantic memory. In: Handbook of functional neuroimaging, Ed 2 (Cabeza R, Kingstone A, eds), pp. 149-190. Cambridge, MA: MIT.

Toglia MP, Battig WF (1978) Handbook of semantic word norms. Hillsdale, NJ: Erlbaum.

Wagner AD, Desmond JE, Demb JB, Glover GH, Gabrieli JDE (1997) Semantic repetition priming for verbal and pictorial knowledge: a functional MRI study of left inferior prefrontal cortex. J Cogn Neurosci 9:714-726.

Wagner AD, Pare-Blagoev EJ, Clark J, Poldrack RA (2001) Recovering meaning: left prefrontal cortex guides controlled semantic retrieval. Neuron 31:329-338.

Wallis JD, Anderson KC, Miller EK (2001) Single neurons in prefrontal cortex encode abstract rules. Nature 411:953-956. 